\title{
Avaliação do Processo de Cura da Borracha Nitrílica (NBR) pela Resina Fenólica através do Cálculo da Constante de Cura
}

\author{
Ana Maria Furtado, Augusto C. C. Peres, Regina C. R. Nunes, Leila L. Y. Visconte \\ Instituto de Macromoléculas Professora Eloisa Mano, UFRJ \\ Cristina R. G. Furtado \\ Instituto de Química, UERJ
}

\begin{abstract}
Resumo: O processo de cura envolve reações de múltiplos mecanismos e sua investigação pode ser feita por meio de várias técnicas. Este trabalho apresenta a comparação entre dois métodos que utilizam parâmetros reométricos para o cálculo da constante cinética de cura $(k)$ relacionada com a cura da borracha nitrílica com a resina fenólica, tendo o policloropreno e o óxido de zinco como sistema ativador. Foi utilizado o planejamento de experimentos fatorial completo $\left(2^{3}+\right.$ ponto central $)$ como base para a preparação das formulações e análise dos resultados. Os resultados obtidos identificaram a influência da temperatura, da quantidade de cada um dos componentes das formulações e da adequação dos métodos de avaliação para o processo de cura da borracha nitrílica pela resina fenólica.
\end{abstract}

Palavras-Chave: Cinética de cura, borracha nitrílica (NBR), resina fenólica, parâmetros reométricos.

\section{Evaluation of Phenolic Resin Vulcanization of Nitrile Rubber (NBR) through Determination of the Cure Constant}

Abstracts: The cure process involves reactions with multiple mechanisms and its investigation can be followed by using different techniques. This work compares two methods in which rheometric parameters are used to calculate the cure rate constant $(\mathrm{k})$, related to the cure of nitrile rubber with phenolic resin and having polychloroprene and zinc oxide as the activators. A complete factorial $\left(2^{3}+\right.$ central point $)$ experimental design was used as base to prepare the formulations and to analyse the results. The results obtained identified the influence of the temperature, the amount of each component in the formulation and the suitability of the evaluation methods for the cure process of nitrile rubber with phenolic resin.

Keywords: Cure kinetics, nitrile rubber (NBR), phenolic resin, rheometric parameters.

\section{Introdução}

O processo de formação de ligações cruzadas em borrachas pode ser promovido por meio de várias substâncias químicas. Quando essa reticulação envolve a utilização de enxofre ou compostos de enxofre, o processo é denominado de vulcanização e, se outros compostos são usados, dá-se também, a denominação de cura. A vulcanização ou cura tem sido, então, alvo de várias tentativas de definição para se encontrar a me- lhor maneira de se descrever as mudanças que ocorrem com os elastômeros durante o processo. Através da vulcanização ou cura, a borracha passa do estado plástico ao estado predominantemente elástico, o que leva ao aumento das forças retrativas e à diminuição da deformação permanente, quando o material é submetido a uma tensão.

As reações envolvidas nesse processo podem ser do tipo adição, substituição, eliminação, ou uma combinação destas. Na investigação dos mecanismos e

Autor para correspondência: Leila L. Y. Visconte, IMA, Universidade Federal do Rio de Janeiro, C. P. 68525, CEP: 21945-970, Rio de Janeiro, RJ. E-mail: lyv@ima.ufrj.br 
estruturas das borrachas vulcanizadas, várias técnicas têm sido utilizadas incluindo análise química, ressonância magnética nuclear de carbono $13\left({ }^{13} \mathrm{C} \mathrm{RMN}\right)$ no estado sólido, determinação da constante cinética, inchamento no equilíbrio, calorimetria diferencial de varredura (DSC), entre outras ${ }^{[1]}$.

Inúmeros aditivos, além dos agentes de vulcanização ou de cura, os quais efetivamente participam do reticulado tridimensional, estão envolvidos no processo de reticulação, cada qual com uma função específica. Os aceleradores reduzem o tempo de vulcanização através do aumento da velocidade da reação entre o enxofre e a borracha ${ }^{[2]}$. Os sistemas de ativação, constituídos normalmente de óxidos metálicos e ácidos graxos para a vulcanização com enxofre e óxidos metálicos e doadores de cloro para cura com resina fenólica, aumentam a eficiência da reação de reticulação, ou seja, incrementam a formação de ligações cruzadas para uma mesma quantidade de agentes de vulcanização ou de cura ${ }^{[3]}$.

A escolha dos componentes de um sistema de cura para uma borracha em particular, depende de suas características e das propriedades requeridas pelo produto final. A borracha nitrílica (NBR) é normalmente vulcanizada pelo sistema convencional enxofre/aceleradores. Porém, a cura com resina fenólica é usada quando se desejam produtos com excelente resistência ao calor, alta resistência a compressões sucessivas e boas propriedades dinâmicas ${ }^{[4]}$. Na cura com resina fenólica a taxa de reação pode ser aumentada pela utilização de ativadores que contenham halogêneos, como os haletos metálicos, policloropreno e polietileno clorossulfonado ${ }^{[4-5]}$, e vários métodos têm sido propostos para o acompanhamento da cinética dessa reação ${ }^{[5-8]}$.

Os métodos baseados nos parâmetros reométricos consideram que a evolução do torque pode ser relacionada diretamente com a formação de ligações cruzadas. Uma cinética de primeira ordem é assumida e a expressão matemática é então expressa pelos valores de torque obtidos durante os primeiros estágios da reação de cura, considerando que a menores conversões, a taxa reflete predominantemente a reação de formação de ligações cruzadas ${ }^{[6]}$.

Entretanto, a taxa máxima de conversão mostrase mais sensível às variações nas quantidades dos ingredientes da formulação que os parâmetros tradicionais fornecidos pelo curômetro ${ }^{[7]}$, e o conhecimento do tempo no qual este máximo ocorre possi- bilita uma determinação mais acurada da constante cinética de cura $k$. Diferentemente do argumento proposto por Chough et al ${ }^{[6]}$, este novo modelo é baseado na taxa decrescente de reação, de modo que somente os dados obtidos após a ocorrência da taxa máxima de cura são considerados. Embora uma cinética de primeira ordem seja predominantemente encontrada para muitos compostos de borracha, este método permite a determinação exata da ordem de reação quando esta é diferente da unidade.

O grande interesse por esta discussão direcionou este trabalho no sentido de investigar a cinética da reação de cura da borracha nitrílica em presença da resina fenólica SP-1045, do policloropreno e do óxido de zinco. $\mathrm{O}$ efeito da quantidade de cada um destes componentes sobre a taxa de reação também foi investigada. Um planejamento de experimentos fatorial completo $\left(2^{3}+\right.$ ponto central) foi elaborado considerando-se estas três variáveis em dois níveis. Os dados reométricos foram manipulados de acordo com os métodos sugeridos por Chough et $\mathrm{a}^{[6]}$ e Dick et $\mathrm{al}^{[7]} \mathrm{e}$ os resultados foram comparados.

\section{Experimental}

Os materiais utilizados foram: borracha nitrílica NP 2021, de médio teor de acrilonitrila (33\%) com $11 \%$ de agente de partição e viscosidade mooney (ML $1+4$ a $\left.100^{\circ} \mathrm{C}\right)=45$, fornecida pela Nitriflex S.A. Industria e Comércio; resina fenólica SP 1045 com teor de metilol $=8,6 \%$, ponto de amolecimento $=$ $92^{\circ} \mathrm{C}$ e viscosidade $\mathrm{GH}=2,74$, fornecida pela Schenectady do Brasil Ltda.; borracha de policloropreno, Neoprene W, com viscosidade mooney $\left(\mathrm{ML} 1+4\right.$ a $\left.100^{\circ} \mathrm{C}\right)=40$, fornecida pela DuPont do Brasil S.A. e óxido de zinco com pureza maior que 99\% fornecido pela Aciquimica Ltda.

A influência da quantidade de cada um dos componentes do sistema de cura foi investigada usandose o planejamento de experimentos fatorial completo mostrado na Tabela 1 .

As misturas foram preparadas a $50^{\circ} \mathrm{C}$ em reômetro de torque Haake, modelo Rheocord RD9000, acoplado a câmara de mistura Reomix 600 e rotores do tipo cam. A velocidade do rotor foi de $20 \mathrm{rpm}$ e o tempo total de mistura foi de 10 minutos. Dados reométricos foram obtidos em reômetro de disco oscilatório Monsanto, modelo $100 \mathrm{~s}$, operando com arco de $3^{\circ}$, nas temperaturas 170,180 e $190^{\circ} \mathrm{C}$. 
Tabela 1. Planejamento fatorial completo $\left(2^{3}+\right.$ ponto central).

\begin{tabular}{lccccccccc}
\hline \multicolumn{1}{c}{ Formulação } & F1 & F2 & F3 & F4(3X) & F5 & F6 & F7 & F8 & F9 \\
\hline Resina Fenólica SP1045 & 1 & -1 & -1 & 0 & 1 & 1 & -1 & 1 & -1 \\
Policloropreno W & -1 & -1 & 1 & 0 & 1 & -1 & -1 & 1 & 1 \\
Óxido de Zinco & -1 & -1 & 1 & 0 & -1 & 1 & 1 & 1 & -1 \\
\hline & & & Em phr & & & & \\
\hline NBR NP 2021 & 11 & 7 & 7 & 9 & 11 & 11 & 7 & 11 & 7 \\
\hline Resina Fenólica SP1045 & 5 & 5 & 15 & 10 & 15 & 5 & 5 & 15 & 15 \\
Policloropreno W & 3 & 3 & 7 & 5 & 3 & 7 & 7 & 7 & 3 \\
Óxido de Zinco & & & & & & & & \\
\hline
\end{tabular}

\section{Resultados e discussão}

A Figura 1 mostra que, nas três temperaturas de cura usadas, o torque máximo é afetado tanto pela concentração da resina como pela concentração do policloropreno, aumentando com o aumento do teor desses aditivos. Ao contrário, o teor de óxido de zinco não tem influência sobre esta propriedade.

O aumento do torque máximo em função do aumento da quantidade de resina já era esperado, visto que este composto funciona como o agente de reticulação. O aumento do torque máximo em função

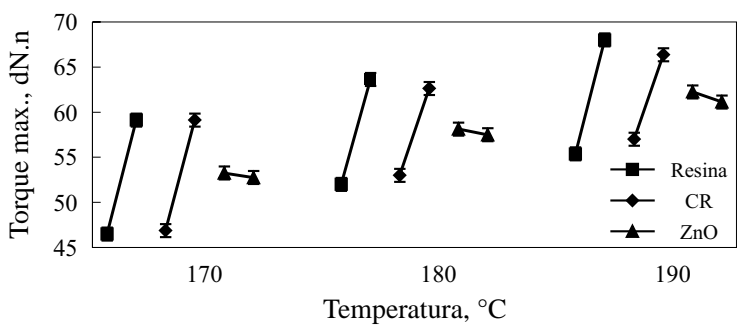

Figura 1. Efeitos dos teores máximo e mínimo de resina fenólica, policloropreno e óxido de zinco nas três temperaturas sobre o torque máximo.

Tabela 2. Formulações com variação dos teores de policloropreno e óxido de zinco (phr).

\begin{tabular}{lcccc}
\hline \multicolumn{1}{c}{ Formulação } & F6 & F8 & F10 & F11 \\
\hline NBR NP 2021 & \multicolumn{4}{c}{100} \\
Resina Fenólica & & & & \\
SP1045 & & & 11 & \\
Policloropreno W & 5 & 15 & 0 & 0 \\
Óxido de Zinco & & 7 & & 0 \\
\hline
\end{tabular}

do aumento da quantidade dos ativadores, também seria esperado, devido ao aumento da eficiência na formação das ligações cruzadas. Este comportamento foi verificado para o policloropreno, porém, não o foi para o óxido de zinco. Para confirmar estes resultados novos experimentos foram realizados. As formulações, nas quais apenas as quantidades de policloropreno e de óxido de zinco foram variadas, são mostradas na Tabela 2.

Os dados de torque máximo obtidos para as formulações da Tabela 2 podem ser vistos nas Figuras 2 e 3. Na Figura 2 verifica-se que quantidades maiores que $5 \mathrm{phr}$ de policloropreno influenciam o torque máximo.

$\mathrm{Na}$ Figura 3 observa-se que os valores de torque máximo são superiores quando o óxido de zinco está presente. A diferença entre os torques máximos das formulações com e sem óxido de zinco diminuem com o aumento da temperatura utilizada na cura anulando-se a $190^{\circ} \mathrm{C}$. Provavelmente, a $190^{\circ} \mathrm{C}$, a energia térmica é suficiente para provocar a produção dos produtos de degradação da resina ${ }^{[4]}$, responsáveis pela reticulação da borracha, em uma quantidade e velocidade compatíveis com as geradas pela presença do óxido de zinco.

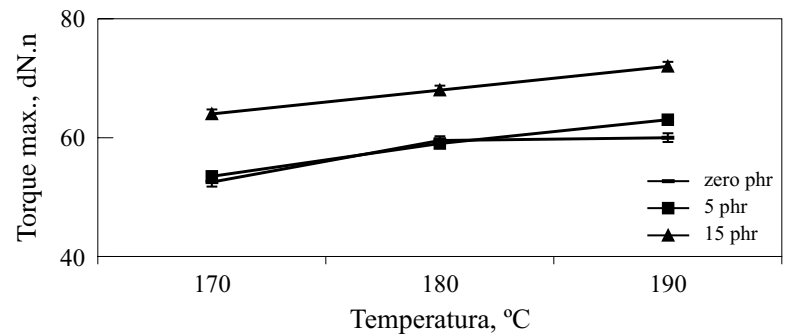

Figura 2. Influência do teor de policloropreno sobre o torque máximo.

Polímeros: Ciência e Tecnologia, vol. 11, ํo 1, p. 9-15, 2001 


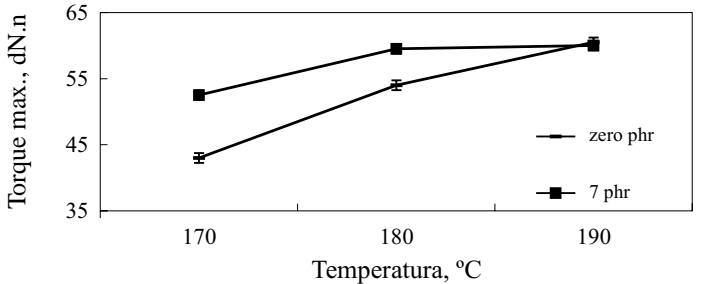

Figura 3. Influência do teor do óxido de zinco sobre o torque máximo.

Comparando-se as Figuras 1 e 3, observa-se que, na primeira, o aumento da quantidade de óxido de zinco de 3 phr (nível -1) para 7 phr (nível 1) não afeta o torque máximo, ao contrário do observado na Figura 3 onde comparou-se a ausência ( 0 phr) com a presença (7 phr) de óxido de zinco. Pode-se deduzir que as quantidades de óxido de zinco empregadas no planejamento estão em excesso.

As constantes cinéticas de cura para a reação de cura foram calculadas utilizando-se dois métodos.

$\mathrm{O}$ primeiro método (Método $\mathrm{A}$ ) considera que os valores de torque, obtidos no reômetro de disco oscilatório durante a cura, estão diretamente relacionados à densidade de ligações cruzadas formadas e que esta reação segue uma cinética de primeira ordem $^{[6]}$. Desse modo, a constante cinética de cura, $k$, pode ser calculada utilizando-se a Equação 1 .

$$
\ln \left[\frac{\text { Torque máx }- \text { Torque min }}{\text { Torque máx - Torque }(\mathrm{t})}\right]=k \mathrm{t}
$$

onde Torque (t) é o valor do torque no tempo igual a t.

Tabela 3. Valores da constante cinética de cura $(k)$, calculados segundo o Método A (ordem da reação $=1$ )

\begin{tabular}{cccc}
\hline \multirow{2}{*}{ Experimento } & \multicolumn{3}{c}{$k, \mathbf{s}^{-\mathbf{1}} \mathbf{1 0 E 0 4}$} \\
\cline { 2 - 4 } & $\mathbf{1 7 0}^{\circ} \mathbf{C}$ & $\mathbf{1 8 0}^{\circ} \mathbf{C}$ & $\mathbf{1 9 0}^{\circ} \mathbf{C}$ \\
\hline 1 & 7,55 & 13,78 & 24,04 \\
2 & 7,95 & 12,16 & 22,97 \\
3 & 12,16 & 22,97 & 32,31 \\
$4-1 \mathrm{r}$ & 8,99 & 16,32 & 28,72 \\
$4-2 \mathrm{r}$ & 9,40 & 17,72 & 31,33 \\
$4-3 \mathrm{r}$ & 9,40 & 18,14 & 31,33 \\
5 & 11,28 & 20,68 & 39,76 \\
6 & 8,74 & 14,77 & 22,97 \\
7 & 8,06 & 13,37 & 19,15 \\
8 & 11,49 & 21,39 & 38,29 \\
9 & 11,70 & 20,40 & 34,46 \\
\hline
\end{tabular}

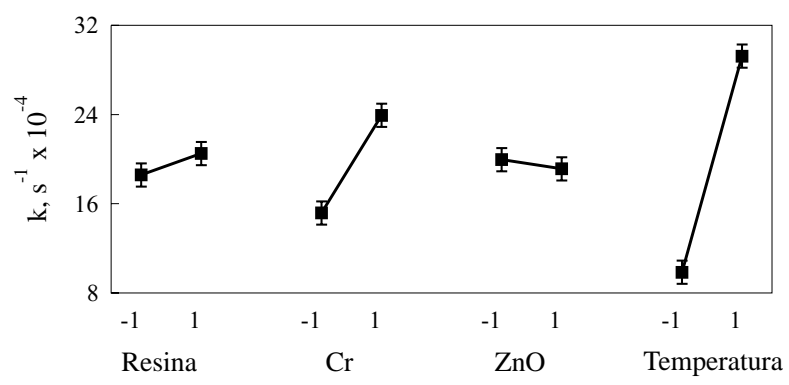

Figura 4. Efeitos dos teores de resina fenólica, policloropreno, óxido de zinco e da temperatura sobre a constante cinética de cura $(k)$ calculada pelo Método A.

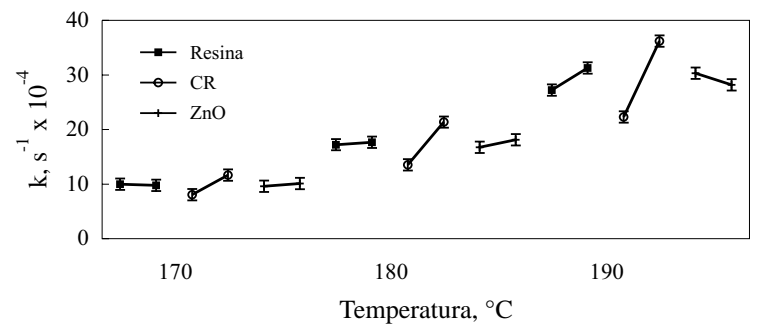

Figura 5. Efeitos dos teores de resina fenólica, policloropreno e óxido de zinco sobre a constante cinética de cura $(k)$ calculada pelo Método A a diferentes temperaturas.

Os valores do torque a 25 e $45 \%$ de conversão foram usados para estimar $k$ e os valores obtidos são apresentados na Tabela 3.

Esses valores foram examinados através da análise de variância, da qual foram obtidas as informações sobre o efeito de cada uma das variáveis e suas interações sobre a constante cinética de cura, mostradas nas Figuras 4 e 5. De acordo com estas Figuras, as variáveis que influenciam $k$ são a temperatura, o teor de policloropreno e a interação entre elas.

Como a cura foi feita nas temperaturas de 170 , 180 e $190^{\circ} \mathrm{C}$, foi investigado o efeito das variáveis sobre $k$ nas três temperaturas. Os resultados são mostrados na Figura 5, comprovando que o policloropreno é a variável mais importante envolvida em cada uma das três temperaturas, sendo que a $190^{\circ} \mathrm{C}$ a resina tem alguma influência sobre $k$, porém em menor intensidade.

No segundo método (Método B) determina-se a ordem da reação usando-se o gráfico de conversão vs. tempo para valores de tempo superiores àquele em que a taxa de conversão é máxima ${ }^{[7]}$. A Equação 2 descreve o comportamento cinético da reação.

$$
\frac{\mathrm{dx}}{\mathrm{dt}}=k^{\mathrm{n}}(1-\mathrm{x})^{\mathrm{n}}
$$


Onde:

$$
\frac{\mathrm{dx}}{\mathrm{dt}}=\text { Taxa de conversão }
$$

$k=$ Constante de velocidade

$\mathrm{n}=$ Ordem da reação

$\mathrm{t}=$ Tempo

Se a reação segue uma cinética de $1^{\mathrm{a}}$ ordem, então $\mathrm{n}=1 \mathrm{e}$, integrando-se a Equação 2 tem-se:

$$
\ln (1-\mathrm{x})=-k(\mathrm{t}-\mathrm{ti})
$$

Onde ti $=$ tempo de indução

Então, plotando-se $\ln (1-\mathrm{x})$ vs. (t-ti) tem-se uma linha reta de coeficiente angular igual a $(-k)$.

Se $\mathrm{n} \neq 1$, a reação seguirá uma cinética de ordem $>1$, quando a concavidade da curva conversão $v s$ tempo for para cima e $<1$ quando a concavidade for para baixo ${ }^{[7]}$. Nestes casos integrando-se a Equação 2 teremos:

$$
\frac{(1-\mathrm{x})^{(1-n)}}{(1-\mathrm{n})}=k^{n}(t-t i)
$$

Então, a ordem de reação é obtida atribuindo-se valores a n na Equação 4 até se obter uma reta com fator de correlação maior que um determinado valor.
Neste trabalho foi arbitrado o valor 0,99 . Os valores de $k$ e $\mathrm{n}$ calculados pela Equação 4 são mostrados na Tabela 4.

Esses valores foram também avaliados pelo método da análise de variância e os seguintes comportamentos foram observados: as variáveis que influenciam significativamente $k$ são a temperatura, o teor de policloropreno, e as interações policloropreno-temperatura e resina fenólica-temperatura, como mostram as Figuras 6 e 7.

Considerando-se agora cada uma das temperaturas em que a cura foi realizada, observa-se que, a 170 e $180^{\circ} \mathrm{C}$, apenas o teor de policloropreno influencia k. A $190^{\circ} \mathrm{C}$ temos a influência da concentração de policloropreno e da resina fenólica conforme mostrado na Figura 7.

A Tabela 5 resume os resultados obtidos nos dois métodos investigados. Observa-se na análise geral, a existência de interação resina-temperatura no Método B, inexistente no Método A.

Por este motivo, foram feitos novos experimentos que pudessem confirmar as conclusões mostradas na Tabela 5. Foram feitas duas novas formulações com NBR e resina fenólica, nas quantidades de 7 e $11 \mathrm{phr}$, curadas a 170,180 e $190^{\circ} \mathrm{C}$. As Figuras 8 e 9 mostram que, no intervalo de temperatura considera-

\begin{tabular}{|c|c|c|c|c|c|c|}
\hline \multirow{2}{*}{ Experimento } & \multicolumn{2}{|c|}{$170^{\circ} \mathrm{C}$} & \multicolumn{2}{|c|}{$180^{\circ} \mathrm{C}$} & \multicolumn{2}{|c|}{$190^{\circ} \mathrm{C}$} \\
\hline & $k, \mathrm{~s}^{-1} 10 \mathrm{E} 04$ & $\mathbf{n}$ & $k, \mathrm{~s}^{-1} 10 \mathrm{E} 04$ & $\mathbf{n}$ & $k, \mathrm{~s}^{-1} 10 \mathrm{E} 04$ & $\mathbf{n}$ \\
\hline 1 & 6,16 & 0,60 & 11,87 & 0,85 & 31,99 & 1,35 \\
\hline 2 & 6,29 & 0,60 & 11,10 & 1,00 & 24,55 & 1,25 \\
\hline 3 & 10,68 & 0,85 & 27,91 & 1,30 & 42,54 & 1,55 \\
\hline $4-1 \mathrm{r}$ & 7,51 & 0,70 & 14,82 & 0,85 & 56,65 & 1,60 \\
\hline $4-2 r$ & 8,50 & 0,75 & 18,19 & 1,15 & 59,84 & 1,75 \\
\hline $4-3 r$ & 9,13 & 0,80 & 23,37 & 1,25 & 51,44 & 1,50 \\
\hline 5 & 12,09 & 0,95 & 26,19 & 1,25 & 92,87 & 1,75 \\
\hline 6 & 7,09 & 0,70 & 20,11 & 1,20 & 34,47 & 1,45 \\
\hline 7 & 6,28 & 0,65 & 11,64 & 0,95 & 20,07 & 1,20 \\
\hline 8 & 10,66 & 0,85 & 24,74 & 1,15 & 79,42 & 1,75 \\
\hline 9 & 12,13 & 0,90 & 28,79 & 1,35 & 58,98 & 1,70 \\
\hline
\end{tabular}
do, não existe interação resina fenólica-temperatura

Tabela 4. Valores da constante cinética de cura $(k)$ e da ordem de reação (n), calculados segundo o Método B. 


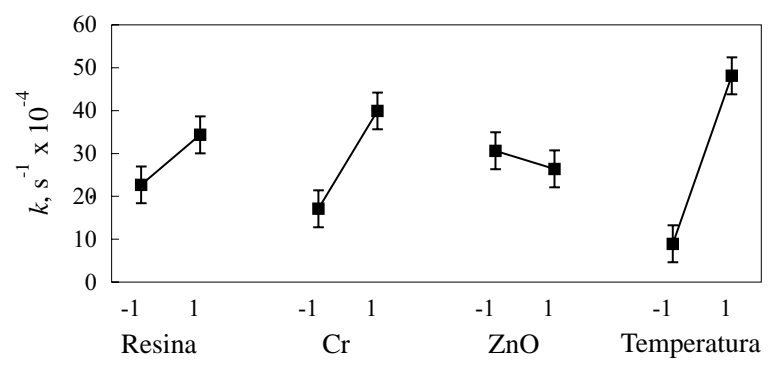

Figura 6. Efeitos dos teores de resina fenólica, policloropreno, óxido de zinco e temperatura sobre a constante cinética de cura $(k)$ calculada pelo Método B.

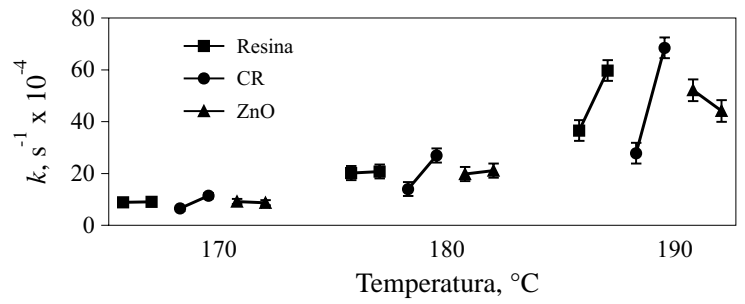

Figura 7. Efeitos dos teores de resina fenólica, policloropreno e óxido de zinco sobre a constante cinética de cura $(k)$, calculada pelo Método B a diferentes temperaturas.

Tabela 5. Resultados das variáveis e interações que afetam $k$ em ordem do grau de importância, obtidos a partir dos Métodos A e B.

\begin{tabular}{ccc}
\hline \multirow{2}{*}{ Análise } & \multicolumn{2}{c}{ Método } \\
\cline { 2 - 3 } & & B \\
& & Temperatura \\
& Temperatura & Policlopreno \\
& Policlopreno & Temperatura / \\
Geral & Interação - & Policroropreno \\
& Temperatura / & Interação \\
& Policroropreno & Temperatura / \\
& & Resina Fenólica \\
& & Policloropreno \\
$170^{\circ} \mathrm{C}$ & Policloropreno & Policloropreno \\
& & \\
\hline \multirow{2}{*}{$180^{\circ} \mathrm{C}$} & Policloropreno & Policloropreno \\
& Policloropreno & Resina fenólica \\
\hline \multirow{2}{*}{$190^{\circ} \mathrm{C}$} & Resina fenólica &
\end{tabular}

já que as duas curvas praticamente se sobrepõem. Esses resultados sugerem que o Método A, em comparação com o Método B, descreve melhor o comportamento cinético deste sistema. Porém, através desta mesma análise, observou-se ainda que a $190^{\circ} \mathrm{C}$ o aumento da quantidade de resina não afetou o valor de $k$, contrariando os resultados anteriormente obtidos e apresentados na Tabela 5.

Em função destes resultados, buscou-se a confirmação da influência das demais variáveis, policloropreno e interação temperatura-policloropreno.

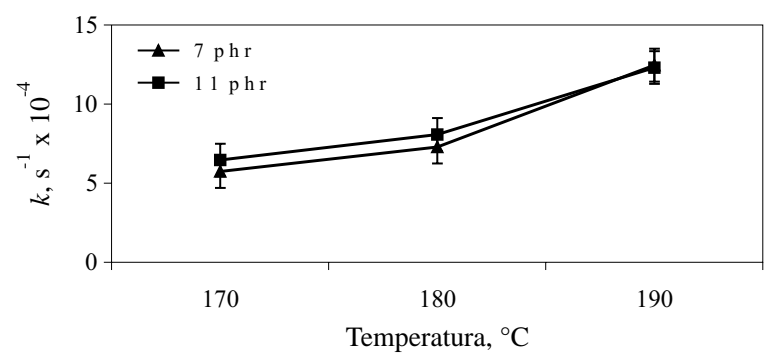

Figura 8. Influência do teor de resina fenólica sobre a constante cinética de cura, calculada pelo Método A, a diferentes temperaturas.

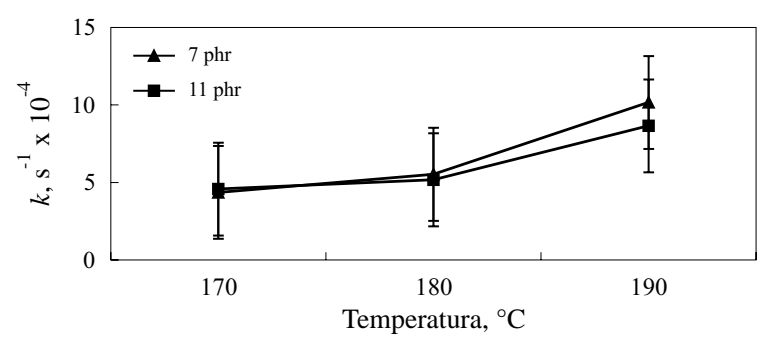

Figura 9. Influência do teor de resina fenólica sobre a constante cinética de cura, calculada pelo Método B, a diferentes temperaturas.

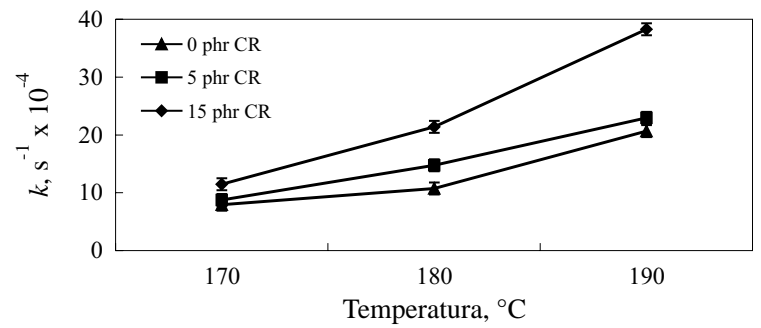

Figura 10. Influência do teor do policloropreno sobre a constante cinética de cura calculada pelo Método A, a diferentes temperaturas (11 phr de resina e $7 \mathrm{phr}$ de $\mathrm{ZnO}$ ).

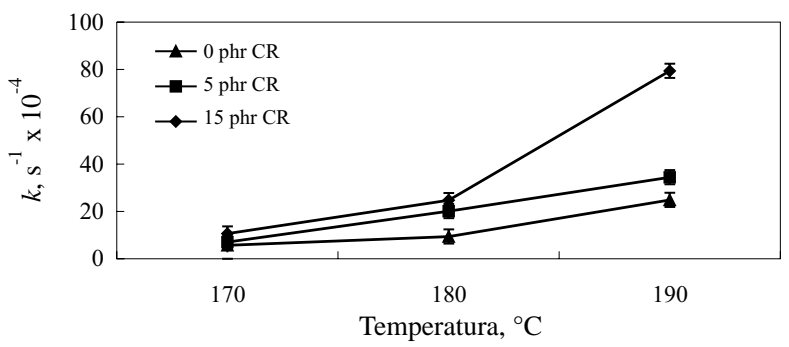

Figura 11. Influência do teor do policloropreno sobre a constante cinética de cura calculada pelo Método B, a diferentes temperaturas (11 phr de resina e $7 \mathrm{phr}$ de $\mathrm{ZnO}$ ).

As Figuras 10 e 11 mostram que o aumento da temperatura e do policloropreno leva a um aumento de $k$ e, pela divergência entre as curvas, que existe a interação temperatura-policloropreno.

\section{Conclusão}

O estudo da cinética de cura das composições 
desenvolvidas, tendo como base duas metodologias (métodos A e B) e da análise estatística foram efetivos na determinação de todos os fatores que influenciam a formação de ligações cruzadas.

A análise experimental dos resultados obtidos pelos métodos acima permitiu afirmar que, apenas os fatores apontados como principais devem ser considerados na utilização dos modelos utilizados neste trabalho.

\section{Referências Bibliográficas}

1. Akiba, M.\& Hashim, A. S. - Prog. Polym. Sci., 22, p.475 (1997).

2. Morton, M. - "Rubber Technology", Robert \& Krieger Publishing Company, Florida (1981).
3. Franta, I. - "Elastomers and Rubber Compounding Materials", Elsevier, Czechoslovakia (1989).

4. van Duin, M.\& Souphanthong, A. - Rubber Chemistry and Technology, 68, p.717 (1995).

5. Konar, B. B. - Journal of Applied Polymer Science, 63, p.233 (1997).

6. Chough, S. H.\& Chang, D.H. - Journal of Applied Polymer Science, 61, p.449 (1996).

7. Dick, J. S.\& Pawlowski, H. - Polymer Testing, 15, p.207 (1996).

8. Volintiru, T.; Ivan, G. \& Buguru, E. - Revue Roumaine de Chimie, 38(3), p.337 (1993).

Recebido: $17 / 10 / 00$

Aprovado: 08/02/01 